\title{
PREOCUPACIONES DE BIG DATA EN MATERIA DE SEGUROS: DE LA SEGMENTACIÓN DE RIESGOS O DE EVENTUALES SITUACIONES DE EXCLUSIÓN O PROBLEMAS DE ACCESO AL MERCADO ASEGURADOR
}

\author{
SOME CONCERNS RELATED TO THE USAGE \\ OF BIG DATA IN INSURANCE MARKETS: \\ SEGMENTATION OF RISKS AND EXCLUSION OF \\ INSURANCE COVERAGE OF SOME POPULATION \\ SECTORS
}

ANDREA LONDOÑO AGUDELO*

El uso de los desarrollos tecnológicos dentro de los mercados financieros, con miras a dar solución a varias problemáticas propias y connaturales de esos mercados ${ }^{1}$, es un asunto que ha despertado especial interés en el transcurso de los últimos años, tanto en los mercados financieros internacionales como en nuestro mercado local.

A la utilización de dichos desarrollos en el mercado financiero se le conoce internacionalmente como FINTECH, refiriéndose con ello,

\footnotetext{
Abogada graduada con honores de la Pontificia Universidad Javeriana de Bogotá, con especialización en seguros de esa misma institución. Ha sido profesora de la Facultad de Derecho de la Pontificia Universidad Javeriana y columnista legal de varios medios locales e internacionales, en la actualidad, además de ser parte del Equipo de Derecho Público de Arrieta Mantilla, es miembro de la lista de secretarios arbitrales del Centro de Arbitraje y Conciliación de la Cámara de Comercio de Bogotá y Miembro de la junta directiva de BMI Colombia Seguros de Vida S.A.. Contacto: andreita_londo@hotmail.com

1 "FinTech' refers to the use of technology to deliver financial solutions (...)". Ver: ARNER, Douglas, et al., Fin Tech, RegTech and Reconceptualization of Financial Regulation. Forthcoming: Northwester Journal of International Law and Business. p. 4.
} 
particularmente, al uso de los mencionados desarrollos con el propósito de lograr innovación en los mercados financieros, a través de nuevos modelos de negocio, de productos y procesos; y mediante la búsqueda de inclusión financiera ${ }^{1}$. A este respecto, por ejemplo, la International Association of Insurance Supervisors ha señalado:

"Following IAIS terminology, this paper uses the term Financial Technologies or "Fintech" to describe technologically enabled financial innovation that could result in new business models, applications, processes, or products with an associated material effect on financial markets and institutions and the provision of financial services. It covers a broad array of technical innovations that are finding their way into the financial industry $(\ldots)^{2}$ ".

Ahora bien, en tratándose de la utilización o aplicación de desarrollos y adelantos tecnológicos en la industria o el mercado asegurador con consiguientes impactos materiales, la doctrina internacional especializada ha denominado a esa circunstancia bajo la expresión INSURTECH ${ }^{3}$, inspirándose, por supuesto, en la expresión utilizada para explicar la misma situación cuando ella acaece o tiene lugar en el marco del sector financiero (FINTECH).

En este sentido, así como hemos sido testigos en los últimos años de un incremento importante y material en la utilización de la tecnología dentro de los mercados financieros locales e internacionales para dar solución, mejorar o hacer más eficiente la actividad adelantada por los intermediarios financieros y las correlativas problemáticas que ella implica o trae consigo, de la misma manera hemos presenciado tal aplicación o utilización en la actividad aseguradora, teniendo en cuenta las características propias y especiales de la misma frente a otro tipo de actividades.

En este orden de ideas, en la actualidad, los reguladores, los super-

1 International Association of Insurance Supervisors, Report of the 24th A2ii - IAIS Consultation Call, Supervising InsurTech, 21 de Septiembre de 2017. p. 1.

2 International Association of Insurance Supervisors, Report of the 24th A2ii - IAIS Consultation Call, Supervising InsurTech, 21 de Septiembre de 2017. p. 1.

3 International Association of Insurance Supervisors, FinTech Developments in the Insurance Industry 21 February 2017, ps. 1-46. 
visores, los agentes del mercado asegurador (compañías de seguros) y los propios consumidores financieros (Tomadores/Asegurados en los Contratos de Seguros) enfrentan la necesidad de entender el contenido y alcance del variado número de actividades que se enmarcan dentro de lo que ha dado en llamarse INSURTECH, así como todo lo que atañe a las consecuencias positivas y negativas de su aplicación.

Dentro de esa gran categoría de actividades que pueden ser catalogadas como INSURTECH, para efectos de este documento, interesa particularmente el fenómeno conocido como Big Data, el cual, a través del diseño e implementación de innovaciones tecnológicas, le permite o permitiría a los agentes, en este caso a las compañías de seguros, la recolección y análisis de una cantidad sumamente grande de información, con el fin de extraer de dicha recolección y análisis variables que resulten de interés a la hora de tomar decisiones relacionadas con la actividad aseguradora por parte de tales agentes. Sobre este aspecto la International Association of Insurance Supervisors ha manifestado:

"The use of Big Data comprises the collection, processing and use of high volumes of different types of data from various sources, using IT tools (powerful processors, software and algorithms), in order to reveal patterns or correlations, generate ideas, solutions or predict certain events or behaviours in a more accurate and timely manner ${ }^{1}$ ".

Teniendo en cuenta la naturaleza de la actividad aseguradora, consistente, en líneas generales, en la aceptación y manejo de riesgos provenientes de terceros (Tomadores/Asegurados), el uso o implementación de herramientas de Biga Data es o puede ser particularmente atractivo, pues si de lo que se trata es de tomar riesgos y administrarlos, el uso de herramientas que permitan recolectar, procesar y analizar información vinculada, directa o indirectamente, con los mismos, es un factor que resulta de indudable interés para esos agentes, esto es, para las aseguradoras.

En la medida en que una compañía de seguros tenga acceso a un número variado y gigante de información asociada en forma, mediata

1 International Association of Insurance Supervisors, FinTech Developments in the Insurance Industry 21 February 2017, p. 33. 
o inmediata, a un riesgo, dispondrá de mejores herramientas para la toma de sus respectivas decisiones de negocio, en tanto dispondrá de un mayor conocimiento del objeto a ser asegurado (Riesgo) y de su Cliente (Tomador/Asegurado), lo cual, evidentemente, la facultará para implementar: (i) Mejores políticas de suscripción y mitigación de riesgos (Contragarantías o Garantías en los Contratos de Seguros), mejores esquemas de tarifación (Pricing); (ii) mejores y más adecuados productos a ser ofrecidos a los consumidores, a la luz de sus necesidades reales y hábitos de consumo y; (iii) mejor y más eficiente diseño y manejo de reclamaciones y políticas antifraude. Lo anterior ha sido expresamente reconocido por la International Association of Insurance Supervisors al establecer que:

"Enhanced data analysis and the availability of unprecedented levels of risk data allow insurers to make much more sophisticated decisions with regards to how to select and manage risks.

\section{$(\ldots)$}

The trends have many potential benefits that align with supervisory objectives. The InsurTech-enabled products may align better with policyholder needs, risk pricing could become more sophisticated and result in insurers managing their exposures betters, and real-time availability of data should inform more timely decisions and facilitate oversight. Digital distribution should help make insurance more affordable, and allow for more targeted products ${ }^{2}$ ".

La utilización de Big Data por parte de las entidades aseguradoras es entonces una herramienta que puede permitir el desarrollo de mercados aseguradores más eficientes y sofisticados y, al mismo tiempo, incontestables ventajas para los consumidores financieros de ese mercado, que muy seguramente podrán obtener, entre otros aspectos, más y mejores productos de seguros y, en algunos casos, precios más accesibles, tal y como ha sido sostenido por importantes organizaciones internacionales, a saber:

2 International Association of Insurance Supervisors, Report of the 24th A2 ii - IAIS Consultation Call, Supervising InsurTech, 21 de Septiembre de 2017. ps. 5 y 7. 
"Digital technology stands to enhance insurance distribution, increase access to insurance, reduce costs and create sufficient scale for a viable business. This positive effect on consumer interaction means that these developments are of particular interest for the inclusive insurance market, as they address some long-standing structural obstacles that have historically caused exclusion from insurance ${ }^{3}$ ".

Podría pensarse que el uso de Big Data por parte de las compañías de seguros lleva necesariamente a mercados que además de caracterizarse por ser más eficientes, también resultan más inclusivos, en la medida en que se permite el acceso a ciertos sectores que pueden haber estado por fuera de la cobertura del negocio asegurador. En efecto, siendo las aseguradoras tomadoras y administradoras de riesgos, una barrera generalmente observada en relación con el acceso de los consumidores a los productos de seguros, consiste en la falta de información de que dispone un asegurador para conocer un riesgo específico, lo que lo lleva a no ofrecer soluciones frente a su transferencia o manejo.

En otras palabras, es frecuente que aun cuando el mercado se enfrente a la necesidad de asegurar o transferir determinados riesgos, en todo caso, las compañías de seguros no estén dispuestas o interesadas en ofrecer mecanismos de cobertura al respecto (Productos de Seguros), por cuanto no tienen la posibilidad de acceder, procesar y analizar información relativa a dichos riesgos y, por tanto, de tomar decisiones racionales en esos frentes.

Así las cosas, resulta indudable que el uso de herramientas de Big Data en el negocio asegurador, arroja, por regla general, consecuencias positivas de cara al propósito superior de inclusión financiera. No obstante, la utilización de las referidas herramientas en tratándose del mercado asegurador, en ciertos casos, puede tener como implicación un efecto contrario al explicado, es decir, un corolario de exclusión financiera.

Si se toma en consideración que a través de las herramientas de Big Data las aseguradoras tienen o podrían tener acceso a un número sin precedentes de información sobre los riesgos a ser asegurados, a su

3 International Association of Insurance Supervisors, Report of the 24th A2ii - IAIS Consultation Call, Supervising InsurTech, 21 de Septiembre de 2017. p. 3. 
respectivo procesamiento y análisis; se tiene que existirán casos en que esa innovación resulte en decisiones de no asegurar riesgos que no son particularmente atractivos, tradicionalmente asociados a sectores que deberían ser objeto de inclusión aseguradora.

La posibilidad de tener mayores variables de decisión racional puede llevar a una aseguradora a establecer que ciertos riesgos (incluidos algunos sectores poblaciones) no son asegurables o que su aseguramiento debe ser extremadamente caro, por cuanto la probabilidad de materialización de un siniestro a ser indemnizado es significativa. En estos escenarios, el propósito público de inclusión financiera puede estar, sin duda, amenazado. Sobre este aspecto la International Association of Insurance Supervisors ha sostenido:

"More available data means risk awareness is increasing, leading to new kind of consumer segmentation (...).

Risk pools may reduce, as advanced analytics may render some segments of the market commercially uninsurable. It may lead to price discrimination and particularly could impact low-income/higher-risk customers ${ }^{4 \prime}$.

En este sentido, no siempre la utilización por parte de las aseguradoras de Big Data cumple con un propósito deseable en materia de inclusión financiera, pues como se ha explicado, también se configuran o pueden configurarse casos en que se concrete exactamente la situación contraria, esto es, que a raíz de procesos de Big Data un asegurador decida que ciertos riesgos no son asegurables en razón de su baja o precaria calidad, o que lo pueden ser en condiciones extremadamente caras ${ }^{\underline{5}}$.

De esta manera, los reguladores y supervisores del mundo deberán diseñar e implementar mecanismos que, sin prohibir el uso de Big Data a las compañías de seguros, dados sus evidentes beneficios, morigeren, atenúen o solucionen problemas como el planteado, especialmente si lo

4 International Association of Insurance Supervisors, Report of the 24th A2ii - IAIS Consultation Call, Supervising InsurTech, 21 de Septiembre de 2017. ps. 6 y 7.

5 "The Mexican supervisor responded that in Non-Life insurance, advanced technology is expected to improve the ability to capture risk. However, in the case of life and health insurance coverages, some technological innovations designed to capture the individualized risk profiles could cause 
que es deseable y debe ser obtenido es la inclusión financiera general.

Los reguladores y supervisores están llamados entonces a cambiar de paradigma, con el fin de diseñar e implementar el balance óptimo entre mercados más eficientes y desarrollados, y la protección debida y suficiente al consumidor financiero ${ }^{6}$.

\section{BIBLIOGRAFÍA}

1. ARNER, Douglas, et al., Fin Tech, RegTech and Reconceptualization of Financial Regulation. Forthcoming: Northwester Journal of International Law and Business.

2. International Association of Insurance Supervisors, Report of the 24th A2ii - IAIS Consultation Call, Supervising InsurTech, 21 de Septiembre de 2017.

3. International Association of Insurance Supervisors, FinTech Developments in the Insurance Industry 21 February 2017.

exclusion and discrimination. Supervisors should keep this in mind when implementing public policies to protect personal data in order to avoid financial exclusion”. En: International Association of Insurance Supervisors, Report of the 24th A2ii - IAIS Consultation Call, Supervising InsurTech, 21 de Septiembre de 2017. p. 12.

6 ARNER, Douglas, et al., Fin Tech, RegTech and Reconceptualization of Financial Regulation. Forthcoming: Northwester Journal of International Law and Business. 